ARTIGO ORIGINAL

ISSN 1677-5090

(C) 2019 Revista de Ciências Médicas e Biológicas

http://dx.doi.org/10.9771/cmbio.v18i3.34115

\title{
Perfil estabilométrico de corredores recreacionais no município de Salvador (BA)
}

\author{
Stabilometric profile of recreational runners in the municipality of Salvador (BA) \\ Renata Santana da Silva Barbosa ${ }^{1}$, Carlos Maurício Cardeal Mendes ${ }^{2}$, Cristiano Sena da Conceição ${ }^{3 *}$ \\ ${ }^{1}$ Fisioterapeuta e Mestranda em Processos Interativos dos Órgãos e Sistemas pela Universidade Federal da Bahia; \\ ${ }^{2}$ Médico e Doutor em Saúde Pública pelo Instituto de Saúde Coletiva da Universidade Federal da Bahia e Prof. \\ Permanente da Pós-graduação em Processos Interativos dos Órgãos e Sistemas - UFBA; ${ }^{3}$ Fisioterapeuta e Doutor \\ em Medicina e Saúde pela Escola Baiana de Medicina e Saúde Pública e Prof. Colaborador do Programa de Pós- \\ Graduação em Processos Interativos dos Órgãos e Sistemas-UFBA
}

\begin{abstract}
Resumo
Introdução: A corrida de rua associada aos benefícios à saúde tem sido a modalidade de escolha para os iniciantes em atividade física. A periodicidade dos treinos, o impacto repetitivo e a presença de terrenos irregulares são características que sobrecarregam o pé e o tornozelo. Para o corredor, uma simples alteração na mecânica do pé repercute no sistema tônico postural e contribui para instabilidade corporal. Dessa forma, os objetivos deste estudo são descrever o perfil estabilométrico e de treinamento de corredores recreacionais e verificar se o tempo e o volume de treinamento estão correlacionados com os dados estabilométricos ligados ao controle postural. Metodologia: A amostra foi composta por 50 corredores recreacionais selecionados por conveniência, com idade entre 18 e 65 anos, de ambos os sexos, sem lesão nos últimos três meses e sem histórico de cirurgias nos membros inferiores. Resultados: Referentes ao perfil de treinamento, os resultados mostraram uma mediana de 2 anos e 6 meses do tempo de treinamento e uma mediana de $20 \mathrm{~km}$ no volume de treinamento semanal. No que se refere ao perfil estabilométrico, a área de oscilação apresentou mediana de $46,7 \mathrm{~mm}^{2}$, a velocidade de oscilação teve média de $8,1 \mathrm{~mm} / \mathrm{s}$ e o desvio padrão foi de 1,2 . Quanto à oscilação, a laterolateral revelou uma mediana de $-1,6 \mathrm{~mm}$, e a anteroposterior uma mediana de $-1 \mathrm{~mm}$. Conclusão: Não houve correlação entre o perfil de treinamento e o perfil estabilométrico. Concluímos que os dados apresentados no perfil do treinamento não estão associados à estabilidade corporal.

Palavras-chave: Corrida. Equilíbrio postural. Treinamento.
\end{abstract}

\begin{abstract}
Introduction: Street running associated with health benefits has been the modality of choice for beginners in physical activity. The periodicity of training, the repetitive impact and the presence of irregular terrain are characteristics that overwhelm the foot and ankle. For the runner, a simple change in the foot mechanics affects the postural tonic system and contributes to body instability. In this way, the goals of this study are to describe the stabilometric and training profile of recreational runners and to verify if the training time and volume are correlated with the stabilometric data related to the postural control. Methodology: The sample consisted of 50 recreational runners, selected for convenience between the ages of 18 and 65, both sexes, without injury in the last 3 months and history of lower limb surgeries. Results: The training profile showed a median of 2 years and 6 months of training time and a median of $20 \mathrm{~km}$ in the weekly training volume. Regarding the stabilometric profile, the area of oscillation with a median of 46.7 $\mathrm{mm}^{2}$ has the oscillation velocity with a mean of $8.1 \mathrm{~mm} / \mathrm{s}$ and a standard deviation of 1.2. As for lateral oscillation with a median of $-1.6 \mathrm{~mm}$, has the anteroposterior oscillation with a median $-1 \mathrm{~mm}$. Conclusion: There was no correlation between the training profile and the stabilometric profile. According to data presented in the training profile, these are not associated to body stability. Keywords: Running. Postural Balance. Training.
\end{abstract}

\section{INTRODUÇÃO}

A prática de atividade física é considerada uma das principais ferramentas para promoção de saúde e prevenção de doenças. Inúmeras evidências apontam que a prática regular de atividade física é capaz de reduzir a morbimortalidade, além de melhorar a função e a qualidade

Correspondente/Corresponding: *Cristiano Sena da Conceição. - End: Universidade Federal da Bahia - PAC - Dpto de Fisioterapia - Tel: (71) 98131-5747 — E-mail: cristianosena@gmail.com. de vida em todo o mundo (EIJSVOGELS et al., 2016; LEE et al., 2012; O'DONOVAN et al., 2017; PIERCY et al., 2018).

Atualmente, a corrida de rua tem sido a modalidade de escolha para os iniciantes. Associada aos benefícios à saúde e aliada à facilidade, essa prática vem atraindo cada vez mais adeptos e se tornando uma modalidade esportiva cada vez mais popular (HULME et al., 2017).

$\mathrm{Na}$ corrida de rua, a periodicidade dos treinos, o impacto repetitivo e a presença de terrenos irregulares são características que sobrecarregam o pé e o tornozelo. 
Assim, para o corredor recreacional (aquele que treina pelo menos uma vez por semana) (GARCIA; SOLER; SALAS, 2017; POPE et al., 2017).

Nessa população, a estabilidade do complexo pé e tornozelo é fundamental para que o adequado controle dinâmico possa suportar as cargas e minimizar a possibilidade de lesão (HULME et al., 2017).

O desempenho de habilidades motoras complexas, como os gestos esportivos, requer grande domínio sobre o equilíbrio. $O$ treinamento (volume de treinamento semanal) e a experiência (tempo de corrida) tornam-se mais eficientes para a ação motora e, por consequência, para o desempenho físico e o equilíbrio entre corredores recreacionais. Um adequado controle do equilíbrio se reflete em sinergias musculares apropriadas, produzindo respostas motoras efetivas, aspectos que são necessários para padrões de movimento com menor potencial lesivo (FIUSA; FRÉZ; PEREIRA, 2015).

A manutenção do equilíbrio em uma postura estática ou dinâmica depende da habilidade do sistema nervoso central em interpretar as informações advindas dos sistemas visual, vestibular e somatossensorial, regulando a atividade muscular por meio de respostas reflexas e voluntárias (REDFERN; YARDLEY; BRONSTEIN, 2015).

Embora a avaliação do equilíbrio seja complexa, uma das técnicas mais utilizadas para aferir a estabilidade corporal é a estabilometria, que consiste na utilização de uma plataforma de pressão sensorizada e acoplada ao solo, capaz de medir a oscilação do corpo por meio da análise do deslocamento do COP (center of pressure) durante o apoio bipodal (BACSI; COLEBATCH, 2015).

Assim, este estudo assume como hipótese que as especificidades ligadas ao tempo e ao volume de treinamento são importantes para um melhor controle postural. Dessa forma, os objetivos deste estudo são descrever o perfil estabilométrico e de treinamento de corredores recreacionais e verificar se o tempo e o volume de treinamento estão correlacionados com os dados estabilométricos ligados ao controle postural.

\section{METODOLOGIA}

\section{Desenho e local do estudo}

Trata-se de um estudo observacional, transversal e descritivo, realizado no período entre abril e setembro de 2018, conduzido no Laboratório da sala 101, no Pavilhão de Aulas do Canela da Universidade Federal da Bahia, situada em Salvador, Bahia.

\section{Critérios de inclusão e exclusão}

A população foi composta por corredores recreacionais da cidade de Salvador (BA). A amostra foi propositiva, selecionada por conveniência em clubes e assessorias esportivas de Salvador, por meio de e-mails, cartazes e redes sociais, contemplando indivíduos sem histórico de lesão nos últimos três meses, com idade entre 18 e 65 anos, de ambos os sexos, que praticam corrida há pelo menos quatro meses sem interrupção, por pelo menos uma vez por semana.

Foram excluídos os indivíduos que apresentaram histórico de cirurgias na extremidade inferior e aqueles que se recusaram a assinar o Termo de Consentimento Livre e Esclarecido.

\section{Coleta de dados}

No primeiro momento, após a assinatura do Termo de Consentimento, os participantes foram entrevistados para coleta de dados demográficos, tempo de prática de corrida e volume de treinamento semanal. Em seguida, foi feito o exame de baropodometria computadorizada, no aparelho da Loran Engineering, que possui eletrodos resistivos e uma superfície ativa de $45 \times 45 \mathrm{~cm}$ para obtenção das variáveis da estabilometria: área e velocidade de oscilação, oscilações laterolateral (LL) e anteroposterior (AP).

Para esse instrumento, foram realizadas 03 coletas de 30 segundos, com o participante em apoio bipodal, com os pés posicionados com largura de base confortável (estática), desde que não ultrapassasse a largura dos ombros e com o olhar fixo em um ponto a uma distância de 3 metros. Assim, foi considerada a terceira medida, já que ela representa um dado mais próximo da média e leva em consideração o efeito da aprendizagem.

\section{Análise de dados}

Os dados coletados foram armazenados no programa Microsoft Excel Starter ${ }^{\circledR} 2016$. As informações foram analisadas através de medidas de frequência simples, de tendência central e de correlação de Spearman no ambiente estatístico do R.

\section{Aspectos éticos}

Todos os participantes foram informados prévia e detalhadamente sobre $a$ avaliação à qual foram submetidos. Receberam o Termo de Consentimento Livre e Esclarecido (TCLE) e, ao concordar com ele, assinaram dando seu consentimento e autorizando sua inclusão na pesquisa. 0 trabalho foi aprovado pelo Comitê de Ética em Pesquisa do Instituto de Ciências da Saúde da Universidade Federal da Bahia, sob o parecer 2.621.166, em abril de 2018.

Ficou esclarecido que o participante teria acesso a todas as informações e poderia desistir da pesquisa ou retirar seu consentimento a qualquer momento, sem prejuízo ou dano, se assim o desejasse. Além disso, foi garantido sigilo absoluto quanto à identificação dos indivíduos, com base nos princípios éticos de confidencialidade e privacidade.

\section{RESULTADOS}

A amostra foi composta de 50 participantes, $62 \%$ do sexo masculino, com idade média de 39,3 anos e desvio 
padrão de 9,5 ; o coeficiente de variação foi de $24,2 \%$. A maioria treinava em assessorias esportivas (82\%).

O tempo de treinamento variou entre 9 meses e 34 anos, com mediana de 2 anos e 6 meses; o volume de treinamento semanal variou de 5 a $60 \mathrm{~km}$, com mediana de $20 \mathrm{~km}$.

No tocante à dominância de membro, $90 \%$ apresentaram dominância à direta e 10\% dominância à esquerda.

Tabela 1 - Perfil demográfico dos participantes de corrida

\begin{tabular}{ll}
\hline Participantes & $\mathrm{n}=50$ \\
\hline Sexo $(\%)^{*}$ & $62 \mathrm{M}$ \\
& $38 \mathrm{~F}$ \\
Tempo de treinamento (meses)** & $30(100)$ \\
Volume de treinamento $(\mathrm{km})^{* * *}$ & $20(75)$ \\
\hline
\end{tabular}

${ }^{*} M=$ masculino/ $F=$ feminino

**Média (desvio-padrão)

***Mediana (coeficiente de variação da mediana-\%)

Fonte: Dados da pesquisa

No que se refere ao perfil estabilométrico, a área de oscilação corporal variou entre 11,1 a $331,5 \mathrm{~mm}^{2}$, com mediana de $46,7 \mathrm{~mm}^{2}$ e coeficiente de variação de $118,2 \%$. A velocidade de oscilação variou de 5,9 a 11,3 $\mathrm{mm} / \mathrm{s}$, com média de $8,1 \mathrm{~mm} / \mathrm{s}$ e desvio padrão de 1,2 . Quanto à oscilação laterolateral, houve variação de $-3,6$ a $5,9 \mathrm{~mm}$, com mediana de $-1,6 \mathrm{~mm}$ e coeficiente de variação da mediana de $-143,7 \%$. Já oscilação anteroposterior variou de $-5,4$ a $1,9 \mathrm{~mm}$, com mediana -1 $\mathrm{mm}$ e coeficiente de variação de $-140 \%$.

A correlação entre o tempo e o volume de treinamento foi de 0,202 , e está representada no gráfico a seguir.

Gráfico 1 - Correlação entre o tempo e volume de treinamento semanal dos corredores*

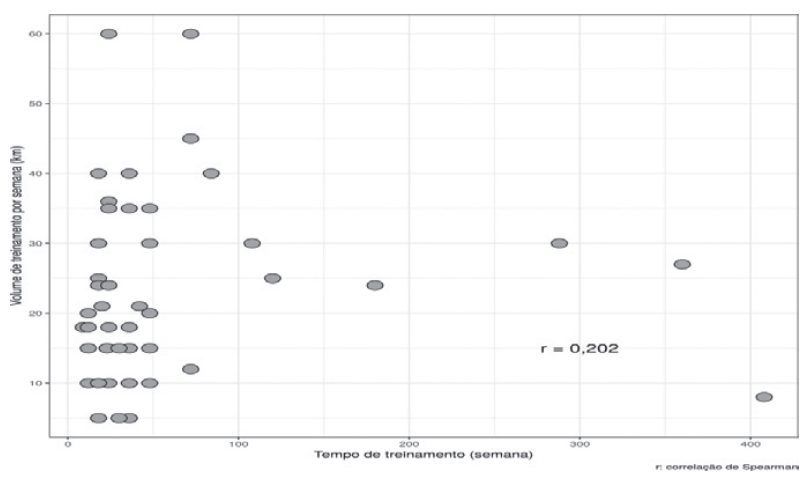

*Índice de correlação de Spearman varia de $r=-1 a$

Fonte: Autoria própria
Tabela 2 - Treinamento e estabilidade corporal*

\begin{tabular}{|c|c|c|c|c|}
\hline \multicolumn{2}{|c|}{ Área de oscilação } & \multirow{2}{*}{$\begin{array}{c}\begin{array}{c}\text { Velocidade de } \\
\text { oscilação }\end{array} \\
0,183\end{array}$} & \multirow{2}{*}{$\begin{array}{c}\begin{array}{c}\text { Oscilação } \\
\text { laterolateral }\end{array} \\
-0,002\end{array}$} & \multirow{2}{*}{$\begin{array}{c}\begin{array}{c}\text { Oscilação } \\
\text { anteroposterior }\end{array} \\
0,078\end{array}$} \\
\hline $\begin{array}{l}\text { Tempo de } \\
\text { treinamento }\end{array}$ & 0,034 & & & \\
\hline $\begin{array}{l}\text { Volume de } \\
\text { treinamento }\end{array}$ & $-0,105$ & 0,091 & 0,243 & 0,027 \\
\hline
\end{tabular}

*Índice de correlação de Spearman varia de $r=-1$ a 1 .

Fonte: Dados da pesquisa

Tabela 3 - Correlação entre medidas de treinamento e estabilidade corporal ajustada pelo IMC*

\begin{tabular}{lcccc}
\hline & $\begin{array}{c}\text { Área de } \\
\text { oscilação }\end{array}$ & $\begin{array}{c}\text { Velocidade de } \\
\text { oscilação }\end{array}$ & $\begin{array}{c}\text { Oscilação } \\
\text { laterolateral }\end{array}$ & $\begin{array}{c}\text { Oscilação } \\
\text { anteroposterior }\end{array}$ \\
\hline $\begin{array}{l}\text { Tempo de } \\
\text { treinamento }\end{array}$ & 0,058 & 0,176 & $-0,005$ & 0,076 \\
$\begin{array}{l}\text { Volume de } \\
\text { treinamento }\end{array}$ & $-0,019$ & 0,037 & 0,238 & 0,074 \\
\hline
\end{tabular}

*IMC (Índice de Massa Corporal) = Peso/Altura $x$ altura. Índice de correlação de Spearman varia de $r=-1$ a 1 .

Fonte: Dados da pesquisa

\section{DISCUSSÃO}

O perfil estabilométrico presente nesta amostra de corredores recreacionais revela intensa variação em área e velocidade de oscilação corporal. Esse resultado pode ser atribuído à grande amplitude na idade. É sabido que a idade interfere no padrão de oscilação e, nesse caso, a área e a velocidade de oscilação tendem a aumentar, ou seja, o controle postural tende a piorar com o avançar da idade. Isso porque a área de oscilação do COP é um índice direto da qualidade do controle postural - quanto maior a variabilidade da oscilação, menos efetivo é o controle postural, com a estabilidade postural reduzida (SARAGIOTTO et al., 2014).

Por outro lado, quando se decompõe a oscilação em deslocamento anteroposterior (AP) e laterolateral (LL), a idade não interferiu no padrão de oscilação, que mostrou uma tendência de oscilação mais frequente na direção lateral esquerda e posterior. É possível que a dominância mais frequente no membro inferior direito tenha contribuído para a maior oscilação no sentido lateral esquerdo. Dixit et al., 2016 encontrou mecanismos de controle postural diferentes na direção AP. Comparados com a direção $\mathrm{LL}$, sugerem que estabilidade postural foi primariamente controlada pela "estratégia do tornozelo" na direção AP e a "estratégia do quadril" na direção LL.

Dentro do contexto do presente estudo, o tempo de prática de corrida não influenciou a estabilidade corporal no apoio bipodal. Esse resultado pode estar ligado ao fato de a corrida ser uma atividade dinâmica, com gestuais amplos, rápidos e em apoio unipodal; nesse caso, as medidas de controle postural estático podem não ser sensíveis a déficits. No entanto, alguns estudos mostraram que corredores treinados aumentaram a estabilidade dinâmica local em comparação com corredores inician- 
tes. $\mathrm{O}$ aumento da experiência de corrida foi fortemente associado ao aumento da estabilidade dinâmica local em tornozelo, joelho e quadril, no plano sagital. Três medidas dinâmicas, que foram relatadas como confiáveis e sensíveis a diferenças de grupos em populações ativas, incluem o Tempo de Estabilização (TTS), o Teste de Equilíbrio das Estrelas por traçado (SEBT) e o Índice Dinâmico de Estabilidade Postural (DPSI). (BUIST et al., 2010; MEARDON; KLUSENDORF; KERNOZEK, 2016).

Outro fato a ser levado em consideração envolve a não especificidade de o treino de corrida envolver treinos de equilíbrio. É possível que a presença desse tipo de treinamento pode ter influenciado no melhor controle postural, como foi verificado em estudo com dançarinos que treinam equilíbrio em condições instáveis (MAHEU et al., 2019).

Diferenças nas habilidades de equilíbrio também foram encontradas entre os controles não treinados e outros grupos de atletas, como judocas, ginastas e acrobatas. Uma questão que emerge é se as mudanças na função de equilíbrio constituem um reflexo das habilidades de equilíbrio intrínseco que predispõem os indivíduos a se especializarem nessas profissões, um resultado do treinamento ou alguma combinação desses fatores (FRANK; PRENTICE; CALLAGHAN, 2019).

Uma importante particularidade pode ser observada quando se compara a distância semanal de treinamento dos participantes deste estudo com a de participantes de outras investigações. Neste caso, a quilometragem do grupo avaliado foi menor quando comparada às distâncias apresentadas na maioria de outras pesquisas também realizadas com corredores (JOHNSTON et al., 2017; TRESCH, 2016).

Essa divergência de resultados pode ser justificada pelo fato de grande parte das pesquisas terem sido conduzidas apenas com maratonistas que requerem um volume de treinamento semanal maior. Acredita-se que essa característica de nosso estudo o torna um dos primeiros a abordar diferentes aspectos relacionados à corrida utilizando uma população de praticantes que não têm o objetivo de correr uma maratona ou uma prova de corrida específica.

\section{Limitações}

No que se refere às limitações de nosso estudo consideramos que não houve grupo de comparação com indivíduos não treinados, tampouco com praticantes de outros esportes. O método de avaliação através da estabilometria é quase estático e pode não representar ou capturar bem a estabilidade dinâmica que está presente em atividades rápidas de apoio unipodal como a corrida.

\section{CONCLUSÃO}

Diante do exposto, foi verificado que os dados apresentados no perfil do treinamento não estão associados ao padrão de oscilação dos COP's. Talvez a presença de um grupo de controle ou de um método dinâmico possa refletir mais adequadamente os efeitos do tempo e do volume de treinamento na estabilidade corporal. Além disso, reiteramos que um corredor mais experiente não necessariamente tem um volume maior de treinamento semanal. No tocante à correlação entre as medidas de treinamento e o perfil estabilométrico, também não houve correlação, indicando que o treinamento da corrida da amostra estudada não esteve associado ao perfil estabilométrico.

\section{REFERÊNCIAS}

BACSI, A. M.; COLEBATCH, J.G. Evidence for reflex and perceptual vestibular contributions to postural control. Exp. brain res., Berlin, v. 160, n. 1, p.22-28, 2015.

BUIST, I. et al. Incidence and risk factors of running-related injuries during preparation for a 4-mile recreational running event. Br. j. sports med., Loughborough, v. 44, n.8, p.598-604, 2010.

DIXIT, S. et al. Analysis of postural control during quiet standing in a population with diabetic peripheral neuropathy undergoing moderate intensity aerobic exercise training: A single blind, randomized controlled trial. Am. j. phys. med. rehabilit., Baltimore, v. 95, n. 7, p.516-524, 2016. DOI:10.1097/PHM.0000000000000426.

EIJSVOGELS, T. M. et al. Exercise at the extremes: the amount of exercise to reduce cardiovascular events. J. Am. Coll. Cardiol., New York, v.67, n.3, p. 316-329, 2016. DOI:10.1016/j.jacc.2015.11.034

FRANK, N. S.; PRENTICE, S. D.; CALLAGHAN, J. P. Local dynamic stability of the lower extremity in novice and trained runners while running intraditional and minimal footwear. Gait posture, Oxford, v. 68, p.50-54, 2019.

FIUSA, J. M.; FRÉZ, A. R.; PEREIRA, W. M. Análise estabilométrica após exercícios proprioceptivos: estudo clínico controlado randomizado. Rev. bras. crescimento desenvolv. hum., São Paulo, v. 25, n. 1, p. $63-67,2015$.

GARCIA, C. A.; SOLER, F. C.; SALAS, C. V. Importancia de la sensibilidad plantar en la regulación del control postural y del movimiento: revisión. Apunts, med. esport., Barcelona, v. 52, n.196, p.149-158, 2017.

HULME, A. et al. Risk and protective factors for middle - and long distance running-related injury. Sports med., Auckland, v.47, n. 5, p. 869-886, 2017.

LEE, I. M. et al. Effect of physical inactivity on major non-communicable diseases worldwide: an analysis of burden of disease and life expectancy. Lancet, London, v. 380, n.9838, p. 219-229, 2012. ISSN: 0140-6736. DOI: 10.1016/S0140-6736(12)61031-9.

JOHNSTON, R. B. et al. Effect of lower extremity muscular fatigue on motor control performance. Med. sci. sports exerc., Madison, v. 30, p.12, p.1703-1707, 2017.

MEARDON, S.; KLUSENDORF, A.; KERNOZEK, T. Influence of injury on dynamic postural control in runners. Int. J. Sports Phys Ther., Bethesda, v.11, n. 3, p. 366-377, 2016.

MAHEU, M. et al. Influence of dance training on challenging postural control task. Gait posture, Oxford, v.69, p. 31-35,2019.

O'DONOVAN, G. et al. Association of "weekend warrior" and other leisure time physical activity patterns with risks for all - cause, cardiovascular disease, and cancer mortality. JAMA intern. med. Chicago, v.177, n.3, p. 335-342, 2017. DOI:10.1001/jamainternmed.2016.8014.

PIERCY, K. L. et al. The physical activity guidelines for Americans. 
JAMA, Chicago, v. 320, n. 19, p. 2020-2028, 2018. DOI:10.1001/jam a.2018.14854.

POPE, M. et al. patial postural control alterations with chronic ankle instability. Gait posture, Oxford, v.34, n.2, p.154-158, 2017.

REDFERN, M.S.; YARDLEY, L.; BRONSTEIN, A. M. Visual influences on balance. J anxiety disord., Elmsford, v.10, p. 85-94, 2015.

SARAGIOTTO, B. T. et al. What are the main risk factors for running-related injuries?. Sports med., Auckland, v. 44, n. 8, p. 1153-1163, 2014.

TRESCH, M. C. A balanced view of motor control. Nat. neurosci., New York, v.10, n.10, p.1227-1228, 2016.

Submetido em: 31/10/2019

Aceito em: 29/11/2019 